Pacific

Journal of

Mathematics

\title{
A NON-ARCHIMEDEAN WAVE EQUATION
}

\author{
ANATOLY N. KochUBEI
}




\title{
A NON-ARCHIMEDEAN WAVE EQUATION
}

\author{
AnATOLY N. Kochubei
}

\begin{abstract}
Let $K$ be a non-Archimedean local field with the normalized absolute value $|\cdot|$. It is shown that a "plane wave" $f\left(t+\omega_{1} x_{1}+\cdots+\omega_{n} x_{n}\right)$, where $f$ is a Bruhat-Schwartz complex-valued test function on $K$ with $\left(t, x_{1}, \ldots, x_{n}\right) \in$ $K^{n+1}$ and $\max _{1 \leq j \leq n}\left|\omega_{j}\right|=1$, satisfies, for any $f$, a certain homogeneous pseudodifferential equation, an analog of the classical wave equation. A theory of the Cauchy problem for this equation is developed.
\end{abstract}

\section{Introduction}

Pseudodifferential equations for complex-valued functions that are defined on nonArchimedean local fields, in particular the field $\mathbb{Q}_{p}$ of $p$-adic numbers, are becoming increasingly important, both in view of rich mathematical structures involved in their studies and due to emerging applications; see the surveys in [Albeverio et al. 2006b; Kochubei 2001; Kochubei and Sait-Ametov 2003; Kozyrev 2004; Varadarajan 2004; Vladimirov et al. 1994; Zuniga-Galindo 2004].

Most studies are of pseudodifferential equations over $\mathbb{Q}_{p}^{n}$ with the symbols $\left|P\left(\xi_{1}, \ldots, \xi_{n}\right)\right|_{p}^{\alpha}$ for $\alpha>0$, where $P$ is a polynomial. The class of elliptic operators correspond to such polynomials $P$ that $P\left(\xi_{1}, \ldots, \xi_{n}\right) \neq 0$ for $\left(\xi_{1}, \ldots, \xi_{n}\right) \neq 0$. An equation of the Schrödinger type is obtained if there is a distinguished variable, say $\xi_{1}$, and $P\left(\xi_{1}, \ldots, \xi_{n}\right)=\xi_{1}-r\left(\xi_{2}, \ldots, \xi_{n}\right)$, where $r$ is a $p$-adic quadratic form. It has been understood also that analogs of parabolic equations are evolution equations with a real time variable and $p$-adic spatial variables (this is connected with probabilistic applications; see [Kochubei 2001] and references therein). It seemed natural to interpret the case where $P\left(\xi_{1}, \ldots, \xi_{n}\right)=\xi_{1}^{2}-r\left(\xi_{2}, \ldots, \xi_{n}\right)$ with an anisotropic quadratic form $r$, as the case of hyperbolic equations. However the results available for this case (see [Kochubei 2001]) are quite scant. In particular, no well-posed problems for such equations have been identified.

In this paper we propose an alternative approach. Instead of a formal resemblance in the definition of an equation, we proceed from its properties. Let us call

MSC2000: primary 11S80, 35S10; secondary 35L99.

Keywords: local field, plane wave, pseudodifferential equation, Cauchy problem.

Partially supported by DFG under Grant 436 UKR 113/87/01, and by the Ukrainian Foundation for Fundamental Research, Grant 14.1/003. 
a function $u\left(t, x_{1}, \ldots, x_{n}\right): \mathbb{Q}_{p}^{n+1} \rightarrow \mathbb{C}$ a plane wave, if, for $\omega_{1}, \ldots, \omega_{n} \in \mathbb{Q}_{p}$ and $\max _{1 \leq j \leq n}\left|\omega_{j}\right|_{p}=1$,

$$
u\left(t, x_{1}, \ldots, x_{n}\right)=f\left(t+\omega_{1} x_{1}+\cdots+\omega_{n} x_{n}\right),
$$

where $f$ belongs to the Bruhat-Schwartz space $\mathscr{D}\left(\mathbb{Q}_{p}\right)$ of test functions (in fact, we will consider not only $\mathbb{Q}_{p}$ but arbitrary non-Archimedean local fields; see below).

We will show that every function (1) is a solution of the equation

$$
D_{t}^{\alpha} u-D_{x}^{\alpha, n} u=0,
$$

where $D^{\alpha}$ is Vladimirov's fractional differentiation operator, that is, a pseudodifferential operator with the symbol $|\xi|_{p}^{\alpha}$, while $D^{\alpha, n}$ is a pseudodifferential operator of $n$ variables with the symbol $\max _{1 \leq j \leq n}\left|\xi_{j}\right|_{p}^{\alpha}$, where $\alpha>0$ is an arbitrary number.

Equation (2) with $n=1$ was mentioned in [Vladimirov 2003] as an example of the following pathology. Consider the equation for a related fundamental solution E:

$$
D_{t}^{\alpha} E-D_{x}^{\alpha, n} E=\delta,
$$

where $E$ belongs to some class of distributions, on which the operators are defined, with the usual relations between them and the Fourier transform. Then, performing the Fourier transform we obtain the contradictory identity

$$
\left(|\tau|_{p}^{\alpha}-\max _{1 \leq j \leq n}\left|\xi_{j}\right|_{p}^{\alpha}\right) \widetilde{E}\left(\tau, \xi_{1}, \ldots, \xi_{n}\right)=1,
$$

where the left side vanishes on the open set

$$
\left\{0 \neq\left(\tau, \xi_{1}, \ldots, \xi_{n}\right) \in \mathbb{Q}_{p}^{n+1}:|\tau|_{p}=\max _{1 \leq j \leq n}\left|\xi_{j}\right|_{p}\right\} .
$$

Therefore the fundamental solution cannot exist, and one cannot expect any reasonable behavior of an inhomogeneous equation associated with (2). On the other hand, the set of solutions of the one-dimensional equation $D_{t}^{\alpha} u=\lambda u$ is infinite-dimensional; see [Kochubei 2001; Vladimirov et al. 1994]. Thus, at first sight, Equation (2) does not look like an evolution equation with "time" variable $t$.

Nevertheless, we will prove here the existence and uniqueness of solutions for some analogs of the Cauchy problem for Equation (2) in the class of radial functions, that is, those depending (in the variable $t$ ) only on $|t|_{p}$. On this class, the operator $D^{\alpha}$ becomes a counterpart of the Caputo-Dzhrbashyan regularized fractional derivative appearing in fractional evolution equations of real analysis; see [Eidelman et al. 2004]. Moreover, the above connection with plane waves, together with the inversion formula for the Radon transform, which is available in the non-Archimedean case too [Chernov 1970; 1972], leads to a formula for solutions and an analog of the Huygens principle. 


\section{Preliminaries}

2.1. Local fields. Let $K$ be a non-Archimedean local field, that is, a nondiscrete totally disconnected locally compact topological field. It is well known that $K$ is isomorphic either to a finite extension of the field $\mathbb{Q}_{p}$ of $p$-adic numbers (if $K$ has characteristic 0), or to the field of formal Laurent series with coefficients from a finite field $\mathbb{F}_{q}$ if $K$ has characteristic $p \neq 0$; in this case $q=p^{v}$ for $v \in \mathbb{N}$. For a summary of main notions and results regarding local fields see, for example, [Kochubei 2001].

Any local field is endowed with an absolute value $|\cdot|$ such that, first, $|x|=0$ if and only if $x=0$, second, $|x y|=|x| \cdot|y|$, and, finally, $|x+y| \leq \max (|x|,|y|)$. Denote $O=\{x \in K:|x| \leq 1\}, P=\{x \in K:|x|<1\}$, and $U=O \backslash P$. $O$ is a subring of $K$ called the ring of integers, $P$ is an ideal in $O$ called the prime ideal; the multiplicative group $U$ is called the group of units. The ideal $P$ contains an element $\beta$ such that $P=\beta O$ (a prime element). The quotient ring $O / P$ is actually a finite field; denote by $q$ its cardinality. We will always assume that the absolute value is normalized, that is, $|\beta|=q^{-1}$. The normalized absolute value $|\cdot|$ takes the values $q^{N}$ for $N \in \mathbb{Z}$.

If $K=\mathbb{Q}_{p}$ ( $p$ is a prime number), that is, a completion of the field $\mathbb{Q}$ of rational numbers with respect to the absolute value

$$
|x|_{p}=p^{-v} \quad \text { for } x=p^{v} \frac{m}{n},
$$

where $\nu, m, n \in \mathbb{Z}$, and $m, n$ are prime to $p$, then $\beta=p$ ( $p$ is seen as an element of $\mathbb{Q}_{p}$ ) and $q=p$ (as a natural number).

Returning to a general local field $K$, denote by $S \subset O$ a complete system of representatives of the residue classes from $O / P$. Then any nonzero element $x \in K$ admits the canonical representation in the form of the convergent series

$$
x=\beta^{-n}\left(x_{0}+x_{1} \beta+x_{2} \beta^{2}+\cdots\right),
$$

where $n \in \mathbb{Z}, \quad|x|=q^{n}, \quad x_{j} \in S$, and $x_{0} \notin P$. For $K=\mathbb{Q}_{p}$, one may choose $S=\{0,1, \ldots, p-1\}$.

The additive group of any local field is self-dual, that is, if $\chi$ is any nonconstant complex-valued additive character of $K$, then any other additive character can be written as $\chi_{a}(x)=\chi(a x)$ for $x \in K$ and some $a \in K$. See [Kochubei 2001] for an explicit description of the character $\chi$ used in harmonic analysis on local fields ("the canonical additive character"). In particular, it is assumed that $\chi$ is a rank zero character, that is, $\chi(x) \equiv 1$ for $x \in O$ and there exists such an element $x_{0} \in K$ that $\left|x_{0}\right|=q$ and $\chi\left(x_{0}\right) \neq 1$.

The duality above is used in the definition of the Fourier transform over $K$. Denoting by $d x$ the Haar measure on the additive group of $K$ (normalized so that 
the measure of $O$ equals 1 ), we write

$$
\widetilde{f}(\xi)=\int_{K} \chi(x \xi) f(x) d x \quad \text { for } \xi \in K,
$$

where $f$ is a complex-valued function from $L_{1}(K)$. As usual, the Fourier transform $\mathscr{F}$ can be extended from $L_{1}(K) \cap L_{2}(K)$ to a unitary operator on $L_{2}(K)$. If $\mathscr{F} f=$ $\widetilde{f} \in L_{1}(K)$, we have the inversion formula $f(x)=\int_{K} \chi(-x \xi) \widetilde{f}(\xi) d \xi$.

Similarly, if $f: K^{n} \rightarrow \mathbb{C}$, we write

$$
\widetilde{f}\left(\xi_{1}, \ldots, \xi_{n}\right)=\int_{K^{n}} \chi\left(x_{1} \xi_{1}+\cdots+x_{n} \xi_{n}\right) f\left(x_{1}, \ldots, x_{n}\right) d x_{1} \ldots d x_{n} .
$$

The inversion formula is then

$$
f\left(x_{1}, \ldots, x_{n}\right)=\int_{K^{n}} \chi\left(-x_{1} \xi_{1}-\cdots-x_{n} \xi_{n}\right) \widetilde{f}\left(\xi_{1}, \ldots, \xi_{n}\right) d \xi_{1} \ldots d \xi_{n} .
$$

2.2. Spaces of test functions and distributions. A function $f: K \rightarrow \mathbb{C}$ is said to be locally constant if there exists such an integer $l$ that for any $x \in K$

$$
f\left(x+x^{\prime}\right)=f(x) \quad \text { if }\left|x^{\prime}\right| \leq q^{-l} .
$$

The smallest number $l$ with this property is called the exponent of local constancy of the function $f$.

Denote by $\mathscr{D}(K)$ the set of all locally constant functions with compact supports. $\mathscr{D}(K)$ is a vector space over $\mathbb{C}$. To furnish it with a topology, consider a subspace $\mathscr{D}_{N}^{l} \subset \mathscr{D}(K)$ of functions supported in the ball

$$
B_{N}=\left\{x \in K:|x| \leq q^{N}\right\} \quad \text { for } n \in \mathbb{Z}
$$

whose exponents of local constancy are $\leq l$.

The space $\mathscr{D}_{N}^{l}$ is finite-dimensional; thus it has a natural topology induced from $\mathbb{C}$. Then we set

$$
\mathscr{D}_{N}=\underset{l \rightarrow \infty}{\lim } \underset{D_{N}^{l}}{\longrightarrow}
$$

and define the topology in $\mathscr{D}(K)$ as the inductive limit topology, that is

$$
\mathscr{D}(K)=\underset{N \rightarrow \infty}{\lim _{N \rightarrow \infty}} \mathscr{D}_{N}
$$

The strong conjugate space $\mathscr{D}^{\prime}(K)$ is called the space of Bruhat-Schwartz distributions.

The operation of the Fourier transform preserves the space $\mathscr{D}(K)$ or $\mathscr{D}\left(K^{n}\right)$ (this property contrasts the Archimedean case). Therefore the Fourier transform of a distribution defined, by duality (just as for distributions from $\mathscr{S}^{\prime}\left(\mathbb{R}^{n}\right)$ ), acts continuously on $\mathscr{D}^{\prime}(K)$ or $\mathscr{D}^{\prime}\left(K^{n}\right)$, respectively. As in the case of $\mathbb{R}^{n}$, there exists 
a well-developed theory of distributions over local fields including such topics as convolution, direct product, homogeneous distributions and so on. Note in particular that a function $|x|^{\alpha-1}$ for $\operatorname{Re} \alpha>0$ admits an analytic continuation in $\alpha$ to a meromorphic distribution

$$
\left\langle|x|^{\alpha-1}, \varphi\right\rangle=\int_{K}|x|^{\alpha-1}(\varphi(x)-\varphi(0)) d x \quad \text { for } \varphi \in \mathscr{D}(K)
$$

and $\operatorname{Re} \alpha>0$ (see [Vladimirov et al. 1994, Section VIII] for $K=\mathbb{Q}_{p}$. The general case is completely similar). See [Albeverio et al. 2006a; Gel'fand et al. 1969; Kochubei 2001; Vladimirov et al. 1994] for further details.

Below we will often use the subspaces of $\mathscr{D}\left(K^{n}\right)$,

$$
\begin{aligned}
& \Psi\left(K^{n}\right)=\left\{\psi \in \mathscr{D}\left(K^{n}\right): \psi(0)=0\right\}, \\
& \Phi\left(K^{n}\right)=\left\{\varphi \in \mathscr{D}\left(K^{n}\right): \int_{K^{n}} \varphi(x) d^{n} x=0\right\},
\end{aligned}
$$

which were introduced in [Albeverio et al. 2006b]. The space $\Phi\left(K^{n}\right)$ is called the Lizorkin space of test functions of the second kind; it is a non-Archimedean counterpart of a space of test functions on $\mathbb{R}^{n}$ proposed by Lizorkin [1963]; see also [Samko 2002]. The conjugate space $\Phi^{\prime}\left(K^{n}\right)$ is called the Lizorkin space of distributions of the second kind. The most important property of these spaces is that the Fourier transform $\mathscr{F}$ is a linear isomorphism from $\Psi\left(K^{n}\right)$ onto $\Phi\left(K^{n}\right)$, thus also from $\Phi^{\prime}\left(K^{n}\right)$ onto $\Psi^{\prime}\left(K^{n}\right)$. At the same time, $\mathscr{F}$ can be considered as a linear isomorphism from $\Phi\left(K^{n}\right)$ to $\Psi\left(K^{n}\right)$.

2.3. Pseudo-differential operators. The simplest and best studied pseudodifferential operator, acting on complex-valued functions over $K$, is the fractional differentiation operator $D^{\alpha}, \alpha>0$, whose deep investigation was initiated by Vladimirov [1994]. It is defined as

$$
\left(D^{\alpha} \varphi\right)(x)=\mathscr{F}^{-1}\left[|\xi|^{\alpha}(\mathscr{F}(\varphi))(\xi)\right](x) \text { for } \varphi \in \mathscr{D}(K) .
$$

Note that $D^{\alpha}$ does not act on the space $\mathscr{D}(K)$ since the function $\xi \mapsto|\xi|^{\alpha}$ is not locally constant. We can assert, for example, that $D^{\alpha}: \mathscr{D}(K) \rightarrow L_{2}(K)$, and the closure of this operator is self-adjoint on $L_{2}(K)$. On the other hand, $D^{\alpha}: \Phi(K) \rightarrow$ $\Phi(K)$ and $D^{\alpha}: \Phi^{\prime}(K) \rightarrow \Phi^{\prime}(K)$; see [Albeverio et al. 2006b].

Similarly, for $x=\left(x_{1}, \ldots, x_{n}\right) \in K^{n}$, set $\|x\|=\max _{1 \leq j \leq n}\left|x_{j}\right|$. The pseudodifferential operator $D^{\alpha, n}: \mathscr{D}\left(K^{n}\right) \rightarrow L_{2}\left(K^{n}\right)$ is given by the expression

$$
\left(D^{\alpha, n} \varphi\right)(x)=\mathscr{F}^{-1}\left[\|\xi\|^{\alpha}(\mathscr{F}(\varphi))(\xi)\right](x) \quad \text { for } \varphi \in \mathscr{D}\left(K^{n}\right) .
$$

We have $D^{\alpha, n}: \Phi\left(K^{n}\right) \rightarrow \Phi\left(K^{n}\right)$ and $D^{\alpha, n}: \Phi^{\prime}\left(K^{n}\right) \rightarrow \Phi^{\prime}\left(K^{n}\right)$.

An important property of these operators is the possibility of getting rid of the Fourier transform and representing them as hypersingular integral operators. For 
any $u \in \mathscr{D}(K)$,

$$
\left(D^{\alpha} u\right)(x)=\frac{1-q^{\alpha}}{1-q^{-\alpha-1}} \int_{K}|y|^{-\alpha-1}(u(x-y)-u(x)) d y ;
$$

see [Kochubei 2001; Vladimirov et al. 1994]. The expression in the right side of (5) makes sense for wider classes of functions, for example, for all bounded locally constant functions.

Similarly, if $u \in \mathscr{D}\left(K^{n}\right)$, then

$$
\left(D^{\alpha, n} u\right)(x)=\frac{1-q^{\alpha}}{1-q^{-\alpha-n}} \int_{K^{n}}\|y\|^{-\alpha-n}(u(x-y)-u(x)) d^{n} y
$$

see [Taibleson 1968; Albeverio et al. 2006b; Vladimirov et al. 1994].

Lemma 1. If $u$ is a bounded locally constant function on $K^{n}$, then the distribution $D^{\alpha, n} u \in \Phi^{\prime}\left(K^{n}\right)$ coincides with the function (6).

Proof. Let $\varphi \in \Phi\left(K^{n}\right)$. Then

$$
\left\langle D^{\alpha, n} u, \varphi\right\rangle=\frac{1-q^{\alpha}}{1-q^{-\alpha-n}} \int_{K^{n}} u(x) d^{n} x \int_{K^{n}} \frac{\varphi(y)-\varphi(x)}{\|x-y\|^{\alpha+n}} d^{n} y .
$$

Let $\theta>0$ be so small that $u(x)=u(y)$ and $\varphi(x)=\varphi(y)$ if $\|x-y\|<\theta$. Denote

$$
C_{\alpha}=\frac{1-q^{\alpha}}{1-q^{-\alpha-n}} \int_{\|y\| \geq \theta} \frac{d y}{\|y\|^{\alpha+n}} .
$$

Then

$$
\begin{aligned}
\left\langle D^{\alpha, n} u, \varphi\right\rangle & =\frac{1-q^{\alpha}}{1-q^{-\alpha-n}} \int_{K^{n}} u(x) d^{n} x \int_{\|x-y\| \geq \theta} \frac{\varphi(y)}{\|x-y\|^{\alpha+n}} d^{n} y-C_{\alpha} \int_{K^{n}} u(x) \varphi(x) d^{n} x \\
& =\frac{1-q^{\alpha}}{1-q^{-\alpha-n}} \int_{K^{n}} \varphi(y) d^{n} y \int_{\|x-y\| \geq \theta} \frac{u(x)-u(y)}{\|x-y\|^{\alpha+n}} d^{n} x=\langle\varphi, \psi\rangle,
\end{aligned}
$$

where $\psi$ is the right-hand side of (6), as desired.

2.4. The Radon transform. Let $\varphi \in \mathscr{D}\left(K^{n}\right)$ for $n \geq 2$. The Radon transform $\check{\varphi}(\xi, s)$, where $\xi \in K^{n}, \xi \neq 0$, and $s \in K$, is defined by the relation

$$
\check{\varphi}(\xi, s)=\int_{\xi \cdot x=s} \varphi(x) d \omega_{\xi, s}(x)
$$

(see [Chernov 1970;1972]), where $\omega_{\xi, s}$ is a measure on the hyperplane $\xi \cdot x=s$ (we write $\left.\xi \cdot x=\xi_{1} x_{1}+\cdots+\xi_{n} x_{n}\right)$ such that, for any $\psi \in \mathscr{D}\left(K^{n}\right)$,

$$
\int_{K} d s \int_{\xi \cdot x=s} \psi(x) d \omega_{\xi, s}(x)=\int_{K^{n}} \psi(x) d x .
$$


The function $\check{\varphi}$ possesses the following properties. It is homogeneous of degree -1 in $\xi$ and $s$, that is, $\check{\varphi}(\sigma \xi, \sigma s)=|\sigma|^{-1} \check{\varphi}(\xi, s)$ for any $\sigma \in K \backslash\{0\}$. Next, $\check{\varphi}(\xi, s)=0$ if the expression $|s| \cdot\|\xi\|^{-1}$ is sufficiently large. The function $\check{\varphi}$ is jointly locally constant in $\xi$ and $s$. Finally, the integral $\int_{K} \check{\varphi}(\xi, s) d s$ does not depend on $\xi$. Note that the above properties of a function of $\xi$ and $s$ are also sufficient for such a function to be the Radon transform of some function $\varphi \in \mathscr{D}\left(K^{n}\right)$.

To find a connection between the Radon and Fourier transforms (similar to the well-known one for the case of $\mathbb{R}^{n}$ [Helgason 1980]), we write

$\widetilde{\varphi}(s \xi)=\int_{K^{n}} \varphi(x) \chi(s(x \cdot \xi)) d^{n} x=\int_{K} d r \int_{\xi \cdot x=r} \varphi(x) \chi(s r) d \omega_{\xi, r}(x)=\int_{K} \chi(s r) \check{\varphi}(\xi, r) d r$,

and it follows from the Fourier inversion formula that

$$
\check{\varphi}(\xi, r)=\int_{K} \chi(-s r) \widetilde{\varphi}(s \xi) d s .
$$

From [Chernov 1970; 1972], the inversion formula for the non-Archimedean Radon transform is

$$
\varphi(x)=\frac{1-q^{n-1}}{\left(1-q^{-1}\right)\left(1-q^{-n}\right)} \int_{\|\eta\|=1}\left\langle|s|^{-n}, \check{\varphi}(\eta, s+\eta \cdot x)\right\rangle d^{n} \eta \quad \text { for } x \in K^{n},
$$

where the distribution $|s|^{-n}$ is understood in the sense of (4). Substituting (4) into (8) and comparing with (5), we can write the inversion formula in the form

$$
\varphi(x)=\left.\frac{1}{1-q^{-1}} \int_{\|\eta\|=1}\left(D_{s}^{n-1} \check{\varphi}(\eta, s+\eta \cdot x)\right)\right|_{s=0} d^{n} \eta .
$$

The identity (9) can be proved directly by substituting (7) and calculating the integrals.

If $n=1$, we define the Radon transform by the formula (7). It is easy to check that the inversion formula (9) remains valid for this case too, in the form

$$
\varphi(x)=\left(1-q^{-1}\right)^{-1} \int_{|\eta|=1} \check{\varphi}(\eta, \eta x) d \eta .
$$

\section{Radial eigenfunctions}

3.1. $L_{2}$-solutions. Let $u(x)=\psi(|x|) \in L_{2}(K)$ be such that

$$
D^{\alpha} u=\lambda u,
$$

where $\lambda=q^{\alpha N}$ for $N \in \mathbb{Z}$ and $u$ is not identically zero.

Let us apply the Fourier transform to both sides of (10). We get

$$
\left(|\xi|^{\alpha}-q^{\alpha N}\right) \widetilde{u}(\xi)=0 \quad \text { for all } \xi \in K .
$$


It follows from (11) that the inequality $\widetilde{u}(\xi) \neq 0$ is possible only for $|\xi|=q^{N}$. Since $u$ is a radial function, $\tilde{u}$ also has this property [Kochubei 2001; Vladimirov et al. 1994]. Therefore

$$
\widetilde{u}(\xi)=\left\{\begin{array}{ll}
c & \text { if }|\xi|=q^{N}, \\
0 & \text { if }|\xi| \neq q^{N},
\end{array} \text { for } c \neq 0 .\right.
$$

By the Fourier inversion and the well-known integration formula (see the two sources above), we get

$$
u(x)= \begin{cases}c q^{N}\left(1-q^{-1}\right) & \text { if }|x| \leq q^{-N} \\ -c q^{N-1} & \text { if }|x|=q^{-N+1} \\ 0 & \text { if }|x|>q^{-N+1}\end{cases}
$$

It is easily seen from (12) or (13) that $u \in \Phi(K)$.

The only radial eigenfunction $u$ with $u(0)=1$ (an analog of the function $t \mapsto e^{-\lambda t}$ for $t \in \mathbb{R})$ corresponds to $c=q^{-N}\left(1-q^{-1}\right)^{-1}$. On the other hand, if $u(0)=0$, then $c=0$.

3.2. Generalized solutions. Let us consider solutions $u \in \Phi^{\prime}(K)$ of Equation (10). It is natural to call a distribution $u \in \Phi^{\prime}(K)$ radial (or spherically symmetric) if, for any $\omega \in K$ with $|\omega|=1$ and any $\varphi \in \Phi(K),\left\langle u, \varphi_{\omega}\right\rangle=\langle u, \varphi\rangle$, where $\varphi_{\omega}(x)=\varphi(\omega x)$. In a similar way, we define a radial distribution from $\Psi^{\prime}(K)$. It is easy to check that the Fourier transform maps a radial distribution from $\Phi^{\prime}(K)$ to a radial distribution from $\Psi^{\prime}(K)$.

Proposition 1. If a radial distribution $u \in \Phi^{\prime}(K)$ satisfies Equation (10), then it coincides, for some $c \in \mathbb{C}$, with the function (13).

Proof. By definition of a generalized solution, we have

$$
\left\langle u, D^{\alpha} \varphi\right\rangle=\lambda\langle u, \varphi\rangle \quad \text { for any } \varphi \in \Phi(K) .
$$

Writing $\varphi=\mathscr{F}^{-1} \psi$, where $\psi \in \Psi(K)$, we see that $\left(D^{\alpha} \varphi\right)(x)=\mathscr{F}_{\xi \rightarrow x}^{-1}\left(|\xi|^{\alpha} \psi(\xi)\right)$.

The function $\xi \rightarrow|\xi|^{\alpha} \psi(\xi)$ belongs to $\Psi(K)$. Therefore, considering $\mathscr{F}_{F}$ as an operator from $\Phi^{\prime}(K)$ to $\Psi^{\prime}(K)$, we may write

$$
\left\langle u, D^{\alpha} \varphi\right\rangle=\left\langle(\mathscr{F} u)(\xi),|\xi|^{\alpha} \psi(\xi)\right\rangle=\left\langle|\xi|^{\alpha}(\mathscr{F} u)(\xi), \psi(\xi)\right\rangle,
$$

so that we come to the equality (11), where this time $\tilde{u}=\mathscr{F} u \in \Psi^{\prime}(K)$, and the multiplication by $|\xi|^{\alpha}-q^{\alpha N}$ is understood in the distributional sense. Thus, for any $\psi \in \Psi(K)$,

$$
\left\langle(\mathscr{F} u)(\xi),\left(|\xi|^{\alpha}-q^{\alpha N}\right) \psi(\xi)\right\rangle=0 .
$$

On the sphere $\left\{\xi \in K:|\xi|=q^{l}\right\}$ for $l \neq N$, the set of functions $\xi \mapsto\left(|\xi|^{\alpha}-\right.$ $\left.q^{\alpha N}\right) \psi(\xi)$ runs the set of restrictions of all the functions from $\Psi(K)$. Therefore 
the restriction of the distribution $\mathscr{F} u$ to such a sphere equals zero, so that $\mathscr{F} u$ is concentrated on the sphere $S_{N}=\left\{\xi \in K:|\xi|=q^{N}\right\}$.

The set of restrictions to $S_{N}$ of functions from $\Psi(K)$ coincides with

$$
\mathscr{D}\left(S_{N}\right)=\underset{l \rightarrow \infty}{\lim } \mathscr{D}_{l}\left(S_{N}\right),
$$

where $\mathscr{D}_{l}\left(S_{N}\right)$ is the set of complex-valued functions on $S_{N}$ with the exponents of local constancy $\leq l$. The space $\mathscr{D}_{l}\left(S_{N}\right)$ is finite-dimensional; its basis can be constructed from the functions $\delta_{\sigma_{0}, \sigma_{1}, \ldots, \sigma_{N+l-1}}(t)$ (here $\sigma_{0}, \sigma_{1}, \ldots, \sigma_{N+l-1} \in S$ and $\left.\sigma_{0} \notin P\right)$, which equal 1 on elements $t \in S_{N}$ with the canonical representations $t=\beta^{-N}\left(\sigma_{0}+\sigma_{1} \beta+\cdots+\sigma_{N+l-1} \beta^{N+l-1}\right)+O\left(\beta^{l}\right)$, and 0 on all other $t \in S_{N}$.

Denote

$$
c_{\sigma_{0}, \sigma_{1}, \ldots, \sigma_{N+l-1}}=\left\langle\mathscr{F} u, \delta_{\sigma_{0}, \sigma_{1}, \ldots, \sigma_{N+l-1}}\right\rangle .
$$

The ratio of any two elements $\beta^{-N}\left(\sigma_{0}+\sigma_{1} \beta+\cdots+\sigma_{N+l-1} \beta^{N+l-1}\right)$ belongs to the group of units $U$. The transformation of one of the functions $\delta_{\sigma_{0}, \sigma_{1}, \ldots, \sigma_{N+l+1}}$ into another (with the same $l$ ) is implemented by multiplying the argument by the appropriate ratio. Since $\mathscr{F} u$ is a radial distribution, we find that $c_{\sigma_{0}, \sigma_{1}, \ldots, \sigma_{N+l-1}}$ depends only on $l$, say $c_{\sigma_{0}, \sigma_{1}, \ldots, \sigma_{N+l-1}}=c_{l-1}^{\prime}$. At the same time,

$$
\sum_{\sigma_{N+l} \in S} \delta_{\sigma_{0}, \sigma_{1}, \ldots, \sigma_{N+l-1}, \sigma_{N+l}}=\delta_{\sigma_{0}, \sigma_{1}, \ldots, \sigma_{N+l-1}},
$$

whence $c_{l-1}^{\prime}=q c_{l}^{\prime}$ and $c_{l}^{\prime}=c_{0}^{\prime} q^{-l}$ for $c_{0}^{\prime} \in \mathbb{C}$. Thus, we have found that

$$
\left\langle\mathscr{F} u, \delta_{\sigma_{0}, \sigma_{1}, \ldots, \sigma_{N+l}}\right\rangle=c_{0}^{\prime} q^{-l}
$$

for all $l$.

Meanwhile, the integral $\int_{|t|=q^{N}} \delta_{\sigma_{0}, \sigma_{1}, \ldots, \sigma_{N+l}}(t) d t$ equals $q^{N-(N+l)-1}=q^{-l-1}$; see [Vladimirov et al. 1994, Section IV]. Together with (14), this shows that the restriction of the distribution $\mathscr{F} u$ to the sphere $S_{N}$ is a constant; outside $S_{N}$, $\mathscr{F} u$ equals 0 . Thus, $\mathscr{F} u$ has the form (12), so that $u$ coincides with the function (13).

\section{Plane waves}

Following a classical pattern we call a function

$$
F(t, x)=f\left(t+\omega_{1} x_{1}+\cdots+\omega_{n} x_{n}\right) \quad \text { for } t \in K \text { and }\left(x_{1}, \ldots, x_{n}\right) \in K^{n},
$$

where $\left\|\left(\omega_{1}, \ldots, \omega_{n}\right)\right\|=1$ and $f \in \mathscr{D}(K)$, a non-Archimedean plane wave.

Proposition 2. For any $\alpha>0$, a non-Archimedean plane wave (15) satisfies the equation

$$
D_{t}^{\alpha} F-D_{x}^{\alpha, n} F=0
$$


Proof. Suppose that $n \geq 2$ (in the case $n=1$ the validity of (16) is checked in a straightforward way). Let us compute $D_{x}^{\alpha, n} F$. By its definition,

$$
\begin{aligned}
\left(D_{x}^{\alpha, n} F\right)(t, x)= & \frac{1-q^{\alpha}}{1-q^{-n-\alpha}} \int_{K^{n}}\left(\max _{j}\left|y_{j}\right|\right)^{-n-\alpha} \\
& \times\left(f\left(t+\sum_{j=1}^{n} \omega_{j} x_{j}-\sum_{j=1}^{n} \omega_{j} y_{j}\right)-f\left(t+\sum_{j=1}^{n} \omega_{j} x_{j}\right)\right) d y_{1} \ldots d y_{n} .
\end{aligned}
$$

Since $\max _{j}\left|\omega_{j}\right|=1$, we can choose an index $j_{0}$ so that $\left|\omega_{j_{0}}\right|=1$. Suppose for simplicity that $\left|\omega_{1}\right|=1$. Let us perform the change of variables

$$
\eta_{1}=\sum_{j=1}^{n} \omega_{j} y_{j}, \quad \eta_{2}=y_{2}, \ldots, \eta_{n}=y_{n} .
$$

Obviously, $\max _{j}\left|\eta_{j}\right| \leq \max _{j}\left|y_{j}\right|$. On the other hand,

$$
y_{1}=\frac{1}{\omega_{1}}\left(\eta_{1}-\omega_{2} \eta_{2}-\cdots-\omega_{n} \eta_{n}\right),
$$

whence $\max _{j}\left|y_{j}\right| \leq \max _{j}\left|\eta_{j}\right|$, so that $\max _{j}\left|y_{j}\right|=\max _{j}\left|\eta_{j}\right|$.

The Jacobian of the transformation $\left(y_{1}, \ldots, y_{n}\right) \mapsto\left(\eta_{1}, \ldots, \eta_{n}\right)$ equals

$$
\left|\begin{array}{cccc}
\omega_{1} & \omega_{2} & \ldots & \omega_{n} \\
0 & & & \\
\vdots & & I_{n-1, n-1} & \\
0 & & &
\end{array}\right|
$$

where $I$ is the appropriate identity matrix. It belongs to $U$. We have

$$
\begin{aligned}
&\left(D_{x}^{\alpha, n} F\right)(t, x)=\frac{1-q^{\alpha}}{1-q^{-n-\alpha}} \int_{K^{n}}\left(\max _{j}\left|\eta_{j}\right|\right)^{-n-\alpha} \\
& \times\left(f\left(t+\sum_{j=1}^{n} \omega_{j} x_{j}-\eta_{1}\right)-f\left(t+\sum_{j=1}^{n} \omega_{j} x_{j}\right)\right) d \eta_{1} \ldots d \eta_{n} \\
&=\frac{1-q^{\alpha}}{1-q^{-n-\alpha}} \int_{K}\left(f\left(t+\sum_{j=1}^{n} \omega_{j} x_{j}-\eta_{1}\right)-f\left(t+\sum_{j=1}^{n} \omega_{j} x_{j}\right)\right) d \eta_{1} \\
& \times \int_{K^{n-1}}\left(\max _{1 \leq j \leq n}\left|\eta_{j}\right|\right)^{-n-\alpha} d \eta_{2} \ldots d \eta_{n} .
\end{aligned}
$$

To compute the integral over $K^{n-1}$, we write it in the form

$$
\int_{K^{n-1}}\left(\max _{1 \leq j \leq n}\left|\eta_{j}\right|\right)^{-n-\alpha} d \eta_{2} \ldots d \eta_{n}=I_{1}+I_{2}
$$


where

$$
\begin{aligned}
& I_{1}=\int_{\max _{2 \leq j \leq n}\left|\eta_{j}\right|<\left|\eta_{1}\right|}\left|\eta_{1}\right|^{-n-\alpha} d \eta_{2} \ldots d \eta_{n}, \\
& I_{2}=\int_{\max _{2 \leq j \leq n}\left|\eta_{j}\right| \geq\left|\eta_{1}\right|}\left(\max _{2 \leq j \leq n}\left|\eta_{j}\right|\right)^{-n-\alpha} d \eta_{2} \ldots d \eta_{n} .
\end{aligned}
$$

It is well known (see, for example, [Taibleson 1968]) that

$$
\int_{\max _{2 \leq j \leq n}\left|\eta_{j}\right|=q^{k}} d \eta_{2} \ldots d \eta_{n}=q^{(n-1) k}\left(1-q^{-n+1}\right) .
$$

Suppose that $\left|\eta_{1}\right|=q^{v}$ for $v \in \mathbb{Z}$. Then

$$
\begin{aligned}
I_{1} & =\left|\eta_{1}\right|^{-n-\alpha} \sum_{k=-\infty}^{\nu-1} q^{(n-1) k}\left(1-q^{-n+1}\right)=\left|\eta_{1}\right|^{-n-\alpha} q^{(n-1)(\nu-1)} \\
& =q^{-(n-1)}\left|\eta_{1}\right|^{-\alpha-1}, \\
I_{2} & =\sum_{k=v}^{\infty} q^{-k(n+\alpha)} q^{(n-1) k}\left(1-q^{-n+1}\right)=\left(1-q^{-n+1}\right) \sum_{k=\nu}^{\infty} q^{-k(\alpha+1)} \\
& =\frac{1-q^{-n+1}}{1-q^{-\alpha-1}}\left|\eta_{1}\right|^{-\alpha-1},
\end{aligned}
$$

so that

$$
\int_{K^{n-1}}\left(\max _{1 \leq j \leq n}\left|\eta_{j}\right|\right)^{-n-\alpha} d \eta_{2} \ldots d \eta_{n}=\frac{1-q^{-n-\alpha}}{1-q^{-\alpha-1}}\left|\eta_{1}\right|^{-\alpha-1} .
$$

Therefore $\left(D_{x}^{\alpha, n} F\right)(t, x)$ equals

$$
\frac{1-q^{\alpha}}{1-q^{-\alpha-1}} \int_{K}\left|\eta_{1}\right|^{-\alpha-1}\left(f\left(t+\sum_{j=1}^{n} \omega_{j} x_{j}-\eta_{1}\right)-f\left(t+\sum_{j=1}^{n} \omega_{j} x_{j}\right)\right) d \eta_{1},
$$

which equals $\left(D_{t}^{\alpha} F\right)(t, x)$. This means that $F$ satisfies Equation (16).

\section{Cauchy problems}

5.1. Applications of the Radon transform. Let $\varphi \in \mathscr{D}\left(K^{n}\right)$. We will look for a solution $F(t, x)$ of Equation (16) satisfying the initial condition

$$
F(0, x)=\varphi(x) \quad \text { for } x \in K^{n},
$$

or the modified initial condition

$$
\left(D_{t}^{n-1} F\right)(0, x)=\varphi(x) \text { for } x \in K^{n} .
$$

Of course, the conditions (17) and (18) coincide if $n=1$.

Let $\check{\varphi}(\xi, s)$ be the Radon transform of the initial function $\varphi$. Denote

$$
\Gamma(t, x, u)=\check{\varphi}(u, t+u \cdot x) \quad \text { for } t \in K, x, u \in K^{n} \text {, and }\|u\|=1 .
$$


Let us consider the functions

$$
\begin{aligned}
& F_{1}(t, x)=\left(1-q^{-1}\right)^{-1} \int_{\|u\|=1}\left(D_{t}^{n-1} \Gamma\right)(t, x, u) d^{n} u, \\
& F_{2}(t, x)=\left(1-q^{-1}\right)^{-1} \int_{\|u\|=1} \Gamma(t, x, u) d^{n} u .
\end{aligned}
$$

Theorem 1. The function $F_{1}(t, x)$ is radial in $t$, is jointly locally constant in $(t, x)$, and is a bounded solution of the Cauchy problem (16), (17). The function $F_{2}(t, x)$ has the same properties, except that it solves the modified Cauchy problem (16), (18).

Proof. It follows from the identity (7) that $\check{\varphi}(\xi, r)$ belongs to $\mathscr{D}(K)$ in $r$ uniformly with respect to $\xi \in K^{n}$ with $\|\xi\|=1$ - there exists a compact set in $K$ outside of which $\check{\varphi}(\xi, \cdot)$ vanishes for all the above $\xi$, and $\check{\varphi}\left(\xi, r+r^{\prime}\right)=\check{\varphi}(\xi, r)$ if $\left|r^{\prime}\right| \leq q^{-l}$, where $l$ does not depend on $\xi$. This means that $\Gamma$ is locally constant in $t$ and $x$ uniformly with respect to $u \in K^{n}$ with $\|u\|=1$. In addition, $\Gamma$ and $D_{t}^{n-1} \Gamma$ are bounded uniformly with respect to $u$. These properties make it possible to change the order of integration while $D_{t}^{\alpha} F_{j}$ and $D_{x}^{\alpha, n}$ are computed. Then Proposition 2 shows that $F_{1}$ and $F_{2}$ satisfy Equation (16). The initial conditions are satisfied due to the Radon inversion formula (9).

To check that $F_{2}(t, x)$ is radial in $t$, we notice that $\check{\varphi}(\omega \xi, \omega s)=\check{\varphi}(\xi, s)$ for $|\omega|=1$, by virtue of the homogeneity property of $\check{\varphi}$. Therefore $\Gamma(\omega t, x, u)=$ $\check{\varphi}(u, \omega t+u \cdot x)=\check{\varphi}\left(\omega^{-1} u, t+\left(\omega^{-1} u\right) \cdot x\right)=\Gamma\left(t, x, \omega^{-1} u\right)$, so that

$$
F_{2}(\omega t, x)=\left(1-q^{-1}\right)^{-1} \int_{\|u\|=1} \Gamma\left(t, x, \omega^{-1} u\right) d^{n} u=F_{2}(t, x) .
$$

Since the operator $D_{t}^{n-1}$ commutes with the operator $f(t) \mapsto f(\omega t)$ with $|\omega|=1$, we find also that $F_{1}$ is radial in $t$.

Let us study the solution $F_{2}(t, x)$ of the modified Cauchy problem (16), (18) in a little greater detail. Using the connection (7) between the Fourier and Radon transforms we get that

$$
\int_{\|u\|=1} \Gamma(t, x, u) d^{n} u=\int_{K} \chi(-s t) d s \int_{\|u\|=1} \chi(-s(u \cdot x)) \tilde{\varphi}(s u) d^{n} u .
$$

Next,

$$
\int_{\|u\|=1} \chi(-s(u \cdot x)) \widetilde{\varphi}(s u) d^{n} u=\int_{K^{n}} \varphi(y) d^{n} y \int_{\|u\|=1} \chi(s(u \cdot(y-x))) d^{n} u .
$$


By the well-known integration formula (see, for example, [Taibleson 1968]),

$$
\int_{\|u\|=1} \chi(s(u \cdot(y-x))) d^{n} u= \begin{cases}1-q^{-n} & \text { if }|s| \cdot\|y-x\| \leq 1 \\ -q^{-n} & \text { if }|s| \cdot\|y-x\|=q, \\ 0 & \text { if }|s| \cdot\|y-x\|>q\end{cases}
$$

so that

$$
\int_{\|u\|=1} \chi(-s(u \cdot x)) \tilde{\varphi}(s u) d^{n} u=\left(1-q^{-n}\right) \int_{\|y-x\| \leq|s|^{-1}} \varphi(y) d^{n} y-q^{-n} \int_{\|y-x\|=q|s|^{-1}} \varphi(y) d^{n} y .
$$

Proposition 3. Suppose that $\varphi(x)=0$ for $\|x\|>q^{N}$, and $\varphi(y)=\varphi(x)$ if $\|y-x\| \leq$ $q^{-v}$ for $v, N \in \mathbb{N}$. Then $F_{2}\left(t+t^{\prime}, x\right)=F_{2}(t, x)$ if $\left|t^{\prime}\right| \leq q^{-v}$, and $F_{2}(t, x)=0$ for $|t|>q^{N+1}$.

Proof. By (19) and (20),

$$
F_{2}(t, x)=\left(1-q^{-1}\right)^{-1} \int_{K} \chi(-s t) R(s, x) d s,
$$

where

$$
R(s, x)=\left(1-q^{-n}\right) \int_{\|y-x\| \leq|s|^{-1}} \varphi(y) d^{n} y-q^{-n} \int_{\|y-x\|=q|s|^{-1}} \varphi(y) d^{n} y .
$$

If $|s| \geq q^{v+1}$, then

$$
\begin{aligned}
R(s, x) & =\varphi(x)\left\{\left(1-q^{-n}\right) \int_{\|y\| \leq|s|^{-1}} d^{n} y-q^{-n} \int_{\|y\|=q|s|^{-1}} d^{n} y\right\} \\
& =\varphi(x)|s|^{-n}\left(\left(1-q^{-n}\right)-q^{-n} \cdot q^{n}\left(1-q^{-n}\right)\right)=0,
\end{aligned}
$$

so that

$$
F_{2}(t, x)=\left(1-q^{-1}\right)^{-1} \int_{|s| \leq q^{\nu}} \chi(-s t) R(s, x) d s,
$$

which implies the required local constancy in $t$.

Let $|t|>q^{N+1}$. Then there exists an element $s_{0} \in K$ with $\left|s_{0}\right|=q^{-N-1}$ such that $\chi\left(s_{0} t\right) \neq 1$. If $\|x\| \leq q^{N}$, then $\varphi(y)=0$ for $\|y-x\|>q^{N}$. Therefore for $|s|<q^{-N}$ the second summand in the right-hand side of (22) equals zero, while the domain of integration in the first summand can be fixed as $\left\{y \in K^{n}:\|y-x\| \leq q^{N}\right\}$ if $|s|<q^{-N}$. Therefore $R(s, x)$ is constant in $s$ on the set $\left\{s \in K:|s|<q^{-N}\right\}$, which implies the equality $R\left(s+s_{0}, x\right)=R(s, x)$ for all the values of $s$. Making in (21) the change of variables $s=s^{\prime}+s_{0}$ we come to the identity $F_{2}(t, x)=\chi\left(s_{0} t\right) F_{2}(t, x)$, which yields the required equality $F_{2}(t, x)=0$.

Note that the local constancy of $F_{2}$ in $t$ may be interpreted as a counterpart of the finite domain of dependence for a classical wave equation: if the initial function $\varphi$ is such that $\varphi(x)=0$ outside some compact set $C \subset K^{n}$, then $F_{2}(t, x)=0$ for 
$x \in K^{n} \backslash C$, at least on some neighborhood of the origin $t=0$. Meanwhile, the fact that $F_{2}(t, x)$ vanishes, as $|t|$ becomes big enough (for a given $\|x\|$ ), resembles the Huygens principle, the existence of the trailing edge of a wave.

5.2. A uniqueness theorem. Here we consider the uniqueness problem in the class of generalized solutions that are radial in $t$.

Denote by $\Phi^{\prime}\left(K, \Phi^{\prime}\left(K^{n}\right)\right)$ the set of distributions over the test function space $\Phi(K)$, with values in $\Phi^{\prime}\left(K^{n}\right)$.

Theorem 2. Let $F \in \Phi^{\prime}\left(K, \Phi^{\prime}\left(K^{n}\right)\right)$ be a generalized solution of the Equation (16), that is, $\left\langle\left\langle F, D_{t}^{\alpha} \varphi_{1}\right\rangle, \varphi_{2}\right\rangle=\left\langle\left\langle F, \varphi_{1}\right\rangle, D_{x}^{\alpha, n} \varphi_{2}\right\rangle$ for any $\varphi_{1} \in \Phi(K), \varphi_{2} \in \Phi\left(K^{n}\right)$. If $F$ is radial in $t$, then $F \in \mathscr{D}\left(K, \Phi^{\prime}\left(K^{n}\right)\right)$. If, in addition, $F(0, x)=0$ or $\left(D_{t}^{n-1} F\right)(0, x)=0$, then $F(t, x) \equiv 0$.

Proof. Denote by $\widetilde{F}(t, \cdot)$ the Fourier transform of $F$ in the variable $x$; as usual, we abuse the notation slightly, writing a distribution in the variable $t$ as a function of $t$. For any $\psi \in \Psi\left(K^{n}\right)$ we have $D_{t}^{\alpha}\langle\widetilde{F}(t, \cdot), \psi\rangle=\left\langle\|\xi\|^{\alpha} \widetilde{F}(t, \xi), \psi(\xi)\right\rangle$. If $\operatorname{supp} \psi \subset$ $S_{N}=\left\{\xi \in K^{n}:\|\xi\|=q^{N}\right\}$ for $N \in \mathbb{N}$, then $D_{t}^{\alpha}\langle\widetilde{F}(t, \cdot), \psi\rangle=q^{\alpha N}\langle\widetilde{F}(t, \cdot), \psi\rangle$.

By Proposition 1, the function $\langle\widetilde{F}(t, \cdot), \psi\rangle$ has the form (13) with $t$ substituted for $x$ and some $c \in \mathbb{C}$. If $\psi \in \Psi\left(K^{n}\right)$, then $\psi$ is a sum of a finite number of functions supported on spheres $S_{N}$. Taking, in particular, $\psi=\widetilde{\varphi}$ for $\varphi \in \Phi\left(K^{n}\right)$, we find that $\langle F(t, \cdot), \varphi\rangle$ belongs to $\mathscr{D}(K)$ in the variable $t$ for any $\varphi \in \Phi\left(K^{n}\right)$.

If $F(0, \cdot)=0$, then also $\widetilde{F}(0, \cdot)=0$. If $\psi \in \Psi\left(K^{n}\right)$ with supp $\psi \subset S_{N}$, then, as we have seen, $\langle\widetilde{F}(t, \cdot), \psi\rangle$ has the form (13), and the assumption $\widetilde{F}(0, \cdot)=0$ implies the equality $c=0$, whence $\langle\widetilde{F}(t, \cdot), \psi\rangle=0$, and $\widetilde{F}(t, \cdot)=0$ (since $\psi$ and $N$ are arbitrary), and $F(t, \cdot)=0$.

Next, if a function $u(t)$ has a form (13), then its Fourier transform has a form (12), and it is easy to find

$$
\left(D^{n-1} u\right)(t)= \begin{cases}c\left(1-q^{-1}\right) q^{N n} & \text { if }|t| \leq q^{-N}, \\ -c q^{N n-1} & \text { if }|t|=q^{-N+1}, \\ 0 & \text { if }|t|>q^{-N+1} .\end{cases}
$$

Repeating the above arguments, we find that the equality $\left.\left(D^{n-1} F\right)(0, x)\right)$ implies $F(t, x) \equiv 0$.

It follows from Lemma 1 that bounded locally constant solutions of Equation (16) are generalized solutions of the class considered in Theorem 2. Therefore the solutions of the Cauchy problems constructed in Theorem 1 are unique in the class of radial in $t$, bounded locally constant functions. It is natural to see such solutions as classical solutions of the non-Archimedean wave equation (16).

5.3. Representation of solutions. Suppose that $\varphi \in \Phi\left(K^{n}\right)$. We will look for a solution belonging to $\Phi(K)$ and radial in $t$, for each $x \in K$, and belonging to $\Phi\left(K^{n}\right)$ 
in $x$, for each $t \in K^{n}$. In this framework, we may use the Fourier transform, but we should not forget to check that the resulting solution indeed satisfies the above requirements.

Let us consider the modified Cauchy problem (16), (18). Suppose that $n \geq 2$. Performing the Fourier transform in $x$ we come to the problem

$$
\begin{aligned}
D_{t}^{\alpha} \widetilde{F}_{2}(t, \xi)-\|\xi\|^{\alpha} \widetilde{F}_{2}(t, \xi) & =0 \\
\left(D_{t}^{n-1} \widetilde{F}_{2}\right)(0, \xi) & =\widetilde{\varphi}(\xi) .
\end{aligned}
$$

As we have seen,

$$
\widetilde{F}_{2}(t, \xi)= \begin{cases}c(\xi)\left(1-q^{-1}\right)\|\xi\| & \text { if }|t| \leq\|\xi\|^{-1} \\ -c(\xi) q^{-1}\|\xi\| & \text { if }|t|=q\|\xi\|^{-1} \\ 0 & \text { if }|t|>q\|\xi\|^{-1}\end{cases}
$$

where $c(\xi) \in \mathbb{C}$ and $c(0)=0$; note that for $\xi=0$ it follows from (23) that $\widetilde{F}_{2}(t, 0)$ is a constant which must equal zero by our assumption that $F_{2} \in \Phi(K)$ in $t$.

Computing $D_{t}^{n-1} \widetilde{F}_{2}$ as above (see the proof of Theorem 2) we find that

$$
\left(D_{t}^{n-1} \widetilde{F}_{2}\right)(t, \xi)= \begin{cases}c(\xi)\left(1-q^{-1}\right)\|\xi\|^{n} & \text { if }|t| \leq\|\xi\|^{-1} \\ -c(\xi) q^{-1}\|\xi\|^{n} & \text { if }|t|=q\|\xi\|^{-1} \\ 0 & \text { if }|t|>q\|\xi\|^{-1}\end{cases}
$$

We find from the initial condition (24) that $c(\xi)=\left(1-q^{-1}\right)^{-1}\|\xi\|^{-n} \widetilde{\varphi}(\xi)$, and we come to the expression

(25) $\widetilde{F}_{2}(t, \xi)=\|\xi\|^{-n+1} b(t \xi) \widetilde{\varphi}(\xi), \quad$ where $b(z)= \begin{cases}1 & \text { if }\|z\| \leq 1, \\ -1 /(q-1) & \text { if }\|z\|=q, \\ 0 & \text { if }\|z\|>q .\end{cases}$

Since $\tilde{\varphi} \in \Psi\left(K^{n}\right)$, it vanishes on a neighborhood of the origin, and it follows from (25) that $\widetilde{F}_{2} \in \Psi\left(K^{n}\right)$ in $\xi$, so that $F_{2} \in \Phi\left(K^{n}\right)$ in $x$. Also, $F_{2}, \widetilde{F}_{2} \in \mathscr{D}(K)$ in $t$ uniformly with respect to $x$ (in the sense of support and local constancy), which permits to interchange operations in different variables. On the other hand, calculating the Fourier transforms, we use (25) to write the solution of $F_{2}(t, x)$ as

$$
F_{2}(t, x)=\left(A * B_{t} * \varphi\right)(x)
$$

where the convolution is taken with respect to $x$, and

$$
\begin{aligned}
& A(x)=\frac{1-q^{-n+1}}{1-q^{-1}}\|x\|^{-1}, \\
& B_{t}(x)=|t|^{-n} \widetilde{b}\left(t^{-1} x\right), \quad \text { where } \widetilde{b}(\zeta)= \begin{cases}\frac{q-q^{n}}{q-1} & \text { if }\|\zeta\| \leq q^{-1} \\
\frac{q}{q-1} & \text { if }\|\zeta\|=1, \\
0 & \text { if }\|\zeta\|>1 .\end{cases}
\end{aligned}
$$


The representation (26) makes it possible, for example, to investigate the dependence $\varphi \mapsto F_{2}(t, \cdot)$ with respect to the $L_{\varkappa}$-norms (for a fixed $t \in K$ ) with $1<\varkappa<\infty$.

Note that

$$
\left\|B_{t}\right\|_{L_{1}\left(K^{n}\right)}=|t|^{-n} \int_{K^{n}}\left|\widetilde{\beta}\left(t^{-1} x\right)\right| d^{n} x=\int_{K^{n}}|\widetilde{\beta}(x)| d^{n} x,
$$

and the Young inequality, together with the commutativity of convolution, gives

$$
\left\|F_{2}(t, \cdot)\right\|_{L_{\varkappa}} \leq C\|A * \varphi\|_{L_{\varkappa}},
$$

where $C$ does not depend on $t$. Applying a result regarding the Riesz potentials from [Taibleson 1968], we find that for $1<\varkappa<n /(n-1)$,

$$
\left\|F_{2}(t, \cdot)\right\|_{L_{\lambda}} \leq C^{\prime}\|\varphi\|_{L_{\varkappa}},
$$

where $\lambda=n \varkappa /(n-\varkappa(n-1))$ and $C^{\prime}$ does not depend on $t$.

For the Cauchy problem (16), (17) (including the case $n=1$ ), we have $F_{1}(t, x)=$ $\left(B_{t} * \varphi\right)(x)$, so that

$$
\left\|F_{1}(t, \cdot)\right\|_{L_{\varkappa}} \leq C\|\varphi\|_{L_{\varkappa}}
$$

for any $\varkappa \in(1, \infty)$, with a constant $C$ independent of $t$.

\section{References}

[Albeverio et al. 2006a] S. Albeverio, A. Y. Khrennikov, and V. M. Shelkovich, "Associated homogeneous $p$-adic distributions", J. Math. Anal. Appl. 313:1 (2006), 64-83. MR 2006g:11234 Zbl 1100.46048

[Albeverio et al. 2006b] S. Albeverio, A. Y. Khrennikov, and V. M. Shelkovich, "Harmonic analysis in the $p$-adic Lizorkin spaces: fractional operators, pseudo-differential equations, $p$-adic wavelets, Tauberian theorems", J. Fourier Anal. Appl. 12 (2006), 393-425. MR 2007g:47076 Zbl 1110.46049

[Chernov 1970] V. G. Chernov, "Homogeneous generalized functions and the Radon transform in a space of rectangular matrices over a continuous locally compact disconnected field.", Dokl. Akad. Nauk SSSR 191 (1970), 540-543. In Russian; translated in Soviet Math. Dokl. 11 (1970), 415-418. MR 41 \#4230 Zbl 0225.46040

[Chernov 1972] V. G. Chernov, "Homogeneous generalized functions and the Radon transform in a space of rectangular matrices over a continuous locally compact nonconnected field", Trudy Sem. Vektor. Tenzor. Anal. 16 (1972), 374-406. In Russian. MR 49 \#3041

[Eidelman et al. 2004] S. D. Eidelman, S. D. Ivasyshen, and A. N. Kochubei, Analytic methods in the theory of differential and pseudo-differential equations of parabolic type, Operator Theory: Advances and Applications 152, Birkhäuser, Basel, 2004. MR 2005m:35002 Zbl 1062.35003

[Gel'fand et al. 1969] I. M. Gel'fand, M. I. Graev, and I. I. Pyatetskii-Shapiro, Representation theory and automorphic functions, W. B. Saunders Co., Philadelphia, 1969. MR 38 \#2093

[Helgason 1980] S. Helgason, The Radon transform, Progress in Mathematics 5, Birkhäuser, Boston, 1980. MR 83f:43012 Zbl 0453.43011 
[Kochubei 2001] A. N. Kochubei, Pseudo-differential equations and stochastics over non-Archimedean fields, Pure and Applied Mathematics 244, Marcel Dekker, New York, 2001. MR 2003b:35220 Zbl 0984.11063

[Kochubei and Sait-Ametov 2003] A. N. Kochubei and M. R. Sait-Ametov, "Interaction measures on the space of distributions over the field of $p$-adic numbers", Infin. Dimens. Anal. Quantum Probab. Relat. Top. 6:3 (2003), 389-411. MR 2004k:81211 Zbl 1053.81067

[Kozyrev 2004] S. V. Kozyrev, " $p$-adic pseudo-differential operators: methods and applications", pp. 154-165 in Избр. вопр. p-адич. мат. физ. и анал., Tr. Mat. Inst. Steklova 245, 2004. MR 2006a:47073 Zbl 1098.47044

[Lizorkin 1963] P. I. Lizorkin, "Generalized Liouville differentiation and the functional spaces $L_{p}{ }^{r}\left(E_{n}\right)$. Imbedding theorems", Mat. Sb. (N.S.) 60 (102) (1963), 325-353. In Russian. MR 27 \#610 Zbl 0134.31703

[Samko 2002] S. G. Samko, Hypersingular integrals and their applications, Analytical Methods and Special Functions 5, Taylor \& Francis, London, 2002. MR 2004a:47057 Zbl 0998.42010

[Taibleson 1968] M. Taibleson, "Harmonic analysis on $n$-dimensional vector spaces over local fields, I: Basic results on fractional integration", Math. Ann. 176 (1968), 191-207. MR 37 \#1984

[Varadarajan 2004] V. S. Varadarajan, "Arithmetic quantum physics: why, what, and whither", pp. 273-280 in Избр. вопр. p-адич. мат. физ. и анал., Tr. Mat. Inst. Steklova 245, 2004. MR 2005g:11262 Zbl 1098.11065

[Vladimirov 2003] V. S. Vladimirov, Таблицы интегралов комплекснозначных функций p-адических аргументов, Современные Проблемы Математики 2, Steklov Institute, Moscow, 2003. MR 2006a:11164 Zbl 1062.11003

[Vladimirov et al. 1994] V. S. Vladimirov, I. V. Volovich, and E. I. Zelenov, p-adic analysis and mathematical physics, Soviet and East European Mathematics 1, World Scientific, River Edge, NJ, 1994. MR 95k:11155 Zbl 0812.46076

[Zuniga-Galindo 2004] W. A. Zuniga-Galindo, "Pseudo-differential equations connected with $p$ adic forms and local zeta functions", Bull. Austral. Math. Soc. 70 (2004), 73-86. MR 2005g:11237 Zbl 1074.46051

Received July 18, 2007. Revised November 5, 2007.

\author{
ANATOLY N. Kochubei \\ INSTITUTE OF MATHEMATICS \\ NATIONAL ACADEMY OF SCIENCES OF UKRAINE \\ TERESHCHENKIVSKA 3 \\ KIEV, 01601 \\ UKRAINE \\ kochubei@i.com.ua
}

